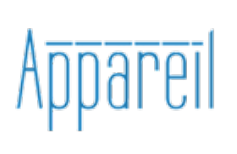

Appareil

18 | 2017

Art et médium 2 : les média dans l'art

\title{
L'exposition comme médium. Quelques observations sur la cybernétisation de l'institution et de l'exposition
}

Yuk Hui et Adeena Mey

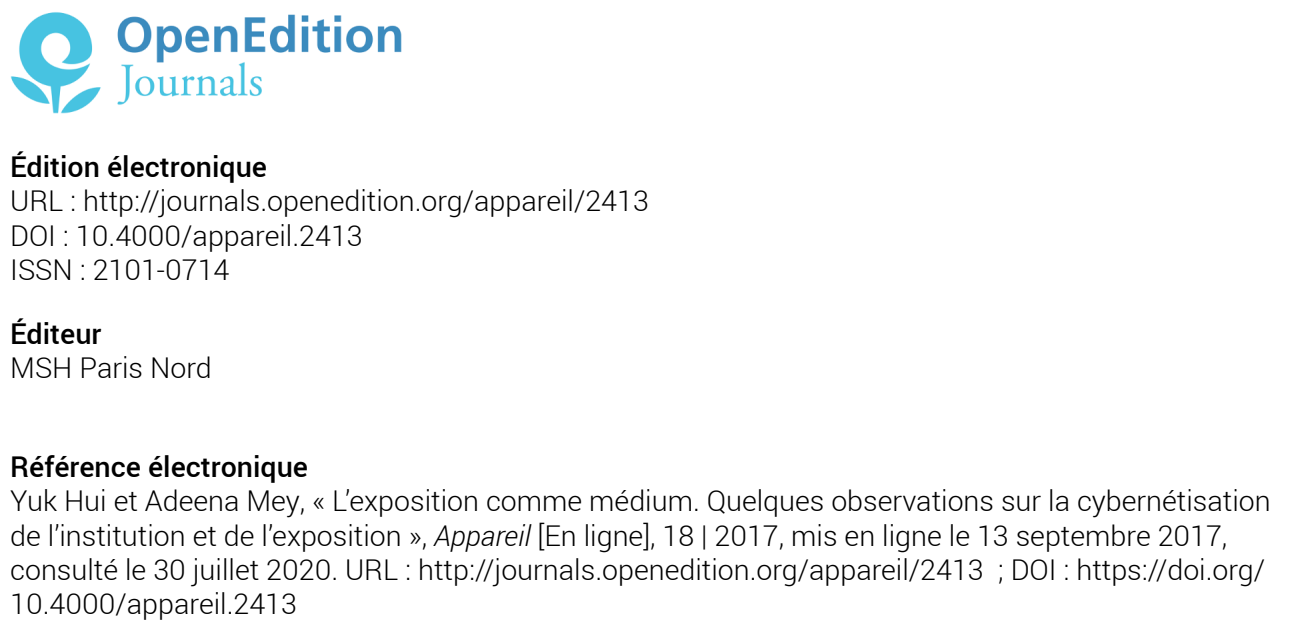

Ce document a été généré automatiquement le 30 juillet 2020.

\section{(c) (i) (9)}

Appareil est mis à disposition selon les termes de la Licence Creative Commons Attribution - Pas d'Utilisation Commerciale - Pas de Modification 4.0 International. 


\title{
L'exposition comme médium. Quelques observations sur la cybernétisation de l'institution et de l'exposition
}

\author{
Yuk Hui et Adeena Mey
}

\section{Introduction}

1 Dans «Qu'appelle-t-on un médium ? », Pascal Krajewski offre un très beau résumé des sens possibles de ce terme, sans pouvoir trancher parmi eux, sur la base d'une discussion détaillée des notions de médium, notamment chez Marshall McLuhan et Régis Debray. À partir de ces deux auteurs, deux approches du médium peuvent être articulées, l'une matérialiste et technique, l'autre plus symbolique et anthropologique.

2 Pour le premier, « le médium est le message ${ }^{1}$ ", parce que le médium parle, c'est-à-dire qu'il porte en lui-même une signification qui a toujours été ignorée ou occultée par le contenu du message qu'il porte. McLuhan propose une notion très générale du médium, affirmant que «tout prolongement technico-technologique de nous-mêmes est un médium ${ }^{2} »$.

3 Pour le second, le médium est «l'ensemble, techniquement et socialement déterminé, des moyens de transmission et de circulation symbolique ${ }^{3}$ ». L'accent est mis sur le symbolique, ce qui restreint de facto toute appréhension du médium à la seule humanité. Il nous semble qu'une généalogie de la relation «symbolique/médium » s'avérerait nécessaire pour développer une notion généralisée et moins anthropocentrique du médium. Une première piste pourrait être l'interprétation du médium comme « milieu », telle que l'a proposée Monique Sicard dans « Du médium au milieu ${ }^{4} »$. Ce dernier désigne littéralement ce qui est au centre, ou ce qui est entre deux choses, connexion qui ouvre la voie à un autre type d'enquête concernant les relations entre tous les êtres. 
Par conséquent, à la lumière de ces premiers défrichages, il nous semble que deux voies s'ouvrent à l'analyse du médium. D'abord, une analyse archéologique et matérialiste, qui concerne la transformation historique d'un standard en un autre, d'une matérialité en une autre et, à leur suite, la transformation sociale et économique; ensuite, une analyse portant sur la communication, l'environnement et l'expérience du sujet et du groupe. Pour parvenir à une définition qui puisse englober les deux sens mentionnés, nous devrons y regarder de plus près. En effet, nous entendons ontologiser le médium au moyen de nouvelles notions fournies par la science et la technologie. Plus précisément, il s'agira de nous distancer de l'approche classique, aristotélicienne, afin de définir le médium comme modulation de tensions, comme système métastable.

Cette définition ontologique nous permettra de proposer une généalogie de l'exposition comme médium à partir de son infrastructure matérielle et comme vecteur de communication, en analysant le processus de cybernétisation et de réinstitutionnalisation de l'exposition tel qu'il se déploie à partir des années 1960 .

\section{Redéfinir le médium}

Pour parvenir à une définition du médium qui risque de paraître étrangère aux historiens de l'art et aux muséologues, nous procéderons en deux temps. Premièrement, nous analyserons la notion de modulation dans le travail des philosophes Gilbert Simondon et Gilles Deleuze. Selon ces deux auteurs, cette notion permet en effet de penser une nouvelle ontologie qui implique également une nouvelle conception de l'esthétique. Dans un deuxième temps, nous articulerons le médium avec le milieu ${ }^{5}$ tel qu'analysé par Georges Canguilhem.

\subsection{Le médium comme modulateur de l'information}

7 Nous proposons de concevoir une définition du médium opératoire et processuelle (c'est-à-dire moins substantialiste). Une pensée substantialiste tend à réduire l'être à une essence, après quoi, celui-ci se ferme sur lui-même. Il s'agit donc d'une pensée statique et rigide qui ignore la dynamique de l'être en cherchant une identité immuable. La pensée scientifique et technique $\mathrm{du} \mathrm{xx}^{\mathrm{e}}$ siècle a ouvert de nouvelles voies pour penser les dynamiques et les processus, et ainsi une ontologie généralisée permettant d'élaborer des théories résolvant l'opposition entre être et devenir, en remontant à leur genèse.

8 La notion de modulation nous permet de penser le médium comme une opération informationnelle et relationnelle. Dans son ouvrage L'individuation à la lumière des notions de forme et d'information, le philosophe Gilbert Simondon a proposé de refonder la question de l'individuation à partir de celle de la modulation, comme alternative à la pensée hylémorphique. L'hylémorphisme se résume à la formule suivante : une forme déjà individualisée impose une essence (eidos) à la matière passive ou inerte ${ }^{6}$. Prenons l'exemple de la fabrication d'une brique, donnant intuitivement l'impression que c'est le moule qui donne sa forme à une matière inerte, dans le cas présent, de l'argile. Simondon critique l'idée selon laquelle la forme du moule est déjà individuée; quant à l'hylémorphisme, il ne permet pas d'expliquer le processus que le philosophe nomme « individuation». Simondon propose donc de comprendre la fabrication d'une brique comme modulation, c'est-à-dire, plus précisément, comme opération informationnelle, 
dans laquelle il ne s'agit plus d'hylémorphisme, mais d'une modulation de l'information en fonction d'une certaine condition matérielle et énergétique : la texture de l'argile, les mains de l'ouvrier, la pression du moule entre les grains, etc. ${ }^{7}$ Cette nouvelle démarche anti-aristotélicienne de Simondon ouvre une voie beaucoup plus large pour comprendre le médium comme une opération qui, bien qu'elle exige un support matériel, résiste à sa réduction à des conditions ou des objets purement matériels. Dans cette perspective, le médium est informationnel et relationnel.

9 La notion d'information joue un rôle central dans la philosophie de Simondon, et par conséquent la notion de forme peut être ressaisie à travers la notion d'information. L'information, selon Simondon, «à la différence de la forme, n'est jamais un terme unique, mais la signification qui surgit d'une disparation ${ }^{8}$ »; une "disparation" émerge de la tension entre deux schèmes ou deux réalités. Nous tentons donc de concevoir le médium comme un processus de traitement des disparations, déclenchant une transindividuation en tant que recherche d'une résolution, pour aboutir à une nouvelle métastabilité.

Dans le contexte de l'art et de l'esthétique, ces disparations peuvent prendre des formes sensibles aux intensités variables, la distinction entre le beau et le sublime d'Emmanuel Kant en est un exemple ${ }^{9}$. La question fondamentale de l'esthétique est celle de la signification que porte le sensible pour celui qui la reçoit. "Être sensible» signifie que ce qui est transmis porte une signification pour le récepteur, opération d'un système auquel il participe. Comme Simondon l'a remarqué :

Être ou ne pas être information ne dépend pas seulement des caractères internes d'une structure; l'information n'est pas une chose, mais l'opération d'une chose arrivant dans un système et y produisant une transformation. L'information ne peut se définir en dehors de cet acte d'incidence transformatrice et de l'opération de réception ${ }^{10}$.

11 Si l'information joue un rôle central, c'est parce qu'elle est nécessaire à l'opération qui produit une transformation au sein d'un être. La modulation est une opération dans laquelle les disparations sont amplifiées ou couplées, faisant émerger une résolution. Nous nous bornerons ici à la modulation du sensible, autrement dit à l'expérience esthétique.

Pareille connexion entre modulation et esthétique se retrouve explicitement dans l'œuvre de Deleuze. En effet, Deleuze reprend la notion simondonnienne de modulation dès son livre Différence et Répétition (1968), et l'applique, comme concept à la fois métaphysique et systématique, à sa relecture de l'histoire de la philosophie ${ }^{11}$. Nous nous référons au cours de Deleuze sur la peinture, dans lequel il propose de comprendre la peinture à la fois comme une modulation de la lumière et comme une modulation de la couleur ${ }^{12}$. Peindre est l'acte de moduler, que ce soit de la couleur, de la lumière ou les deux. Selon la lecture qu'en fait Deleuze, Cézanne emploie la technique de la modulation de la couleur, non pas de la lumière, la différence consistant dans le fait que l'artiste juxtapose des couleurs (un «nouveau régime de la couleur» dit Deleuze), et ce faisant, produit une figure, de la « ressemblance non similaire ${ }^{13}$ ». Nous pouvons comprendre cette ressemblance non similaire ce que Simondon nomme disparation, qui est l'information.

13 La modulation de la peinture a pour but de produire un espace signal qui s'adresse à des spectateurs; autrement dit, la création artistique tend vers un médium communicationnel, car une œuvre est un moyen d'individuation à la fois psychique et 
collective, entre le créateur et son public. Deleuze montre que ce sont les techniques de modulation qui définissent les espaces signaux, selon des techniques qui varient en fonction des cultures : il existe un espace signal grec, un espace signal byzantin, un espace signal égyptien. L'emploi du mot «signal» chez Deleuze requiert une certaine précaution de notre part, tout comme l'usage qui en est fait en sciences de la communication et en sémiotique, où il se trouve alors chargé d'une signification technique. Les signaux n'étant pas séparés de l'espace, la modulation esthétique peut donc être conçue comme visant à produire un «espace signal " comme "partage du sensible ${ }^{14} »$. L'espace signal est aussi un espace de significations, c'est-à-dire qu'il module les sens. Ainsi, les régimes du sensible comme disparations (comme dans le cas de la peinture), permettent au sujet de les intérioriser effectivement et de construire son propre monde.

\subsection{Le médium comme intermédiaire avec le milieu}

Dans Le vivant et son milieu, Canguilhem retrace la généalogie du terme « milieu » et les diverses nuances que ses usages ont pu prendre depuis le xvIII ${ }^{e}$ siècle. Dès le début de son texte, il note que le milieu «est en train de devenir un mode universel et obligatoire de saisie de l'expérience et de l'existence des êtres vivants ${ }^{15}$ ». Son enquête révèle la dimension profondément dialectique qui s'ouvre entre le milieu et le vivant qui l'habite. Si un premier mouvement insiste sur l'adaptation du vivant à son milieu, donné, conditionnant - Canguilhem remarque aussi comment le milieu est, en retour, façonné par le vivant. Ainsi le milieu n'est plus seulement à comprendre comme l'environnement donné et brut, mais comme le résultat d'une élaboration par le vivant de son habitat.

Il y a donc un phénomène d'adaptation du vivant, redoublé par une opération d'élaboration du milieu - dans un circuit bouclé. Nous proposons d'appeler «médium ", ce qui justement opère dans ce processus entre le vivant et son milieu. Le médium indique comment le milieu influe sur le vivant et aussi, comment le vivant opère sur son milieu pour le façonner selon ses besoins.

Dans le cas de l'homme, le milieu ne peut être réduit à un environnement géographique ou physico-chimique. Il contient aussi toutes les sphères représentationnelles et symboliques - de sorte que le vivant, quand il s'agit de l'homme, doit aussi être compris selon ces deux dimensions: il est environnement physique et sphère sémiotique, chargée de sens. Le médium, au cœur du processus de co-détermination entre le milieu et l'homme, aura donc un effet sur les plans physique et symbolique. Le monde humain est riche de médiums qui permettent la modulation de processus d'intériorisation du milieu selon des règles préalables.

Si l'environnement brut donné n'a plus cours et doit être remplacé par l'idée de milieu, résultat de l'action de l'homme sur l'environnement, cette transformation passe par la soumission de l'environnement à une normativité ${ }^{16}$. Dès lors, l'activité humaine se définit notamment par sa capacité à modifier certaines normativités et à rompre avec elles en faveur d'une autre normativité compatible avec la vie. C'est là que l'on trouve aussi les actes créatifs qui transforment le milieu de manière radicale, et qu'on désigne par création artistique ou invention. Ces actes peuvent également être des actes puissants qui agissent à une échelle géographique ${ }^{17}$. Aussi, Canguilhem écrit-il : «en 
effet, le milieu du vivant est aussi l'œuvre du vivant qui se soustrait ou s'offre électivement à certaines influences ${ }^{18}$. "

Le milieu propre à l'homme n'est pas celui de l'animal, car l'homme est capable de modifier son milieu en lui attribuant des significations symboliques, ainsi que des fonctions pragmatiques ${ }^{19}$. À la différence du milieu d'un animal, celui de l'homme convoque une médiation technique et symbolique comportant deux temporalités, l'une historique et anthropologique, l'autre médiatique et phénoménologique. La richesse du médium est ce qui distingue le monde animal du monde humain, l'homme étant le seul animal capable de créer un milieu technique agissant sur l'environnement extérieur : ainsi, nous avons un monde de symboles en-dessus du monde de signes. Le monde des animaux, lui, ne contient que des signaux d'où découlent des significations. Ces signaux constituent ce que Deleuze nomme un « espace signal ». Par conséquent l'adaptation de l'homme à son milieu, contrairement à celle de l'animal, se modalise bel et bien sous deux aspects: un aspect zoologique d'adaptation aux contraintes artificielles et naturelles de l'environnement, et une intériorisation de la sémiose généralisée et signifiante qui le baigne.

Entre l'intériorisation symbolique et le milieu extérieur, c'est-à-dire entre le subjectif et l'objectif, on trouve un intermédiaire, et c'est ce que nous continuons de nommer médium. Ce dernier n'est pas donné, ni comme ce que l'on désigne d'habitude par «nature » ou « environnement », ni comme ce qui va être anticipé et interprété par le sujet-humain ou le sujet-animal. Le médium, tel que nous l'entendons, relèverait plutôt d'une opération faisant médiation entre deux réalités, agissant comme un mécanisme de modulation.

Le monde humain est riche de médiums par lesquels le processus d'intériorisation peut être modulé à partir de règles préalables. Comme mentionné plus haut, selon Canguilhem, la transformation de l'environnement en milieu résulte d'une soumission à un type de normativité. Dans leur évolution, les médiums produisent constamment des dynamiques symboliques, qui à leur tour reconfigurent les médiums dont l'intériorisation permet à chacun de créer sa propre vision du monde.

21 C'est à ce sens-là que tend notre proposition de théorisation de l'exposition comme médium. Plus précisément, l'exposition n'est ni un environnement objectif, ni un milieu déjà construit une fois pour toutes, mais un modulateur évoluant selon une technicité. Dans cette perspective, l'exposition est aussi un milieu spécifique à l'homme car il est un espace foisonnant de sens et de signaux. Elle n'est pas donnée immédiatement comme un paysage quelconque, mais est construite pour optimiser et amplifier certains signaux. Elle est véritablement une modulation chargée de sens, d'énergie et de potentiel. Nous explorons, plus bas, le moment de cybernétisation du musée et des expositions comme une mutation à la fois technique et éco-sociale.

\section{Le médium Exposition}

22 Nous pouvons à présent nous pencher sur la période 1960-1970, moment critique pour la pratique artistique comme pour l'institution muséale. Effectivement, à cette époque, se développent des pratiques artistiques dites "intermédiatiques" et d'autres démarches artistiques conceptuelles, processuelles ou encore performatives, regroupées sous le poncif de "dématérialisation de l'art ${ }^{20}$ ". Mais ce sont moins les reconfigurations du médium de l'art comme manifestant un prétendu tournant vers 
l'« intermédia ${ }^{21}$ » ou vers la «condition post-médium ${ }^{22}$ » qui nous intéressent ici que celles du médium de l'exposition, faisant apparaittre de nouvelles formes d'intégration de ces pratiques au sein des institutions et des expositions.

Posons que l'exposition est un médium qui, par exemple, se situe entre une série d'œuvres d'art et les schèmes psycho-sociaux du public - la première constituant $l e$ milieu, et les seconds sa représentation. Elle module, à travers des objets concrets, parergonaux, et engage la représentation de chaque visiteur en modulant les informations comme la lumière, les couleurs, les sons et les gestes, et en produisant in fine un effet psychique et social. À partir de cette conceptualisation, nous pourrions commencer l'enquête sur l'infrastructure de l'exposition à partir de la notion d'information, en la considérant comme un système autorégulateur.

Il convient donc de se détacher de l'image de l'exposition comme espace fermé accueillant des œuvres d'art et des visiteurs. Nous la comprenons plutôt comme un système dont la croissance peut être exponentielle, traversant les institutions : elle crée un espace-signe non limité aux objets dans une salle, s'incarnant plutôt dans une réticulation d'information à l'intérieur des salles d'exposition, et se déployant dans une constellation inter-institutionnelle voire transnationale. Il nous semble que ce développement passe par deux voies, reprenant chacune des deux modulations du terme médium telles qu'on vient de les proposer : l'exposition, comme reconstruction d'un nouvel environnement, passant par une réticulation institutionnelle et transnationale; et la «cybernétisation » de l'exposition, c'est-à-dire sa conceptualisation comme « centre de communication ».

\subsection{Le « milieu » muséal : Réticulation institutionnelle}

Nous analyserons tout d'abord la réticulation de l'exposition, car elle nous semble constituer un premier niveau au sein de cette dynamique de reconfiguration. Bien qu'ayant accueilli les diverses expériences du modernisme, jusque dans les années 1960, le modèle du musée demeurait celui établi au XIX ${ }^{e}$ siècle, fondé sur une épistémologie rationaliste établissant la classification comme modalité d'accès au savoir, corollaire du sujet voyant et étant vu. C'est dans une volonté de rupture avec ce paradigme - où l'exposition est conçue comme simple réceptacle d'objets offerts à la contemplation de ce sujet - que la possibilité d'un nouveau type de musée est débattue dans les années 1960-1970. Cette dynamique est contemporaine et entre en interaction avec d'autres transformations. D'une part, l'élargissement des pratiques artistiques (les « expanded arts ", synthétisés dans le diagramme produit par George Maciunas en $1966^{23}$ ) et d'autre part, le développement du régime intermédiatique des arts qui vient battre en brèche le principe moderniste de la spécificité du médium défini par Greenberg. En outre, cette expansion est réalisée dans les pratiques dites de «critique institutionnelle ${ }^{24}$ » dont le mouvement au dehors - de l'institution, du musée, de l'exposition - établit ces derniers comme matériau même de la production artistique, l'objet de la critique devenant le médium même par lequel est formulée cette critique.

L'institution produit une certaine structure du désir, elle active un certain espace dans lequel signifiants et désirs circulent, et en ce sens, il est tout aussi futile de rêver d'un espace complètement désinstitutionnalisé, comme d'une institution qui fonctionne - car, comme Deleuze et Guattari le diront plus tard, les structures " dures » et segmentaires ne fonctionnent que sur la base de leurs 'lignes de fuite' ou de leurs fuites, parce que quelque chose leur échappe, irrévocablement. Une 
société ou une institution ne tient que grâce à ce qui fuit, de même que la ligne de fuite est le seul point de déterritorialisation d'une structure donnée ${ }^{26}$.

C

De plus, la déterritorialisation implique en même temps une reterritorialisation qui vise à une métastabilité. Les lignes de fuite cherchent toujours les nouvelles réticulations circulant entre le public, les institutions sociales, financières, politiques, etc. En ce sens, l'exposition est détachée d'un lieu et des objets, c'est-à-dire qu'il ne s'agit pas simplement d'un médium produisant une médiation entre des objets et des spectateurs, mais qu'elle se répand hors d'elle-même pour occuper un nouveau territoire.

Pour le curateur Paul O'Neill, l'exposition comme médium résulte notamment d'une « convergence entre pratiques artistiques et curatoriales ». O'Neill situe la fonction de médium de l'exposition entre deux pôles, celui de la communication d'une part, et celui de sa faculté à déterminer des pratiques sociales de l'autre. Il se base sur les analyses du critique d'art Bruce Ferguson qui affirme que :

[C'est par rapport au] site de présentation plutôt qu'en relation au moment de production d'une œuvre d'art individuelle que l'exposition génère de telles structures hiérarchiques, pour produire des formes générales et spécifiques de communication. [...] l'exposition artistique temporaire est devenue le médium principal de distribution et de réception de l'art, et dès lors, l'agent principal du débat et de la critique, concernant tous les aspects des arts visuels ${ }^{27}$.

Par ailleurs, cette période de redéfinition du musée et de l'exposition se caractérise aussi par des phénomènes d'hybridation ou d'indifférenciation des modalités de présentation de l'art, la galerie ne représentant alors qu'un espace parmi d'autres où artistes et curateurs expérimentent diverses possibilités de présentation de l'art. Une liste non-exhaustive composant cette topologie multiple pourrait inclure la salle de cinéma et la temporalité linéaire à laquelle est assigné le spectateur, le festival comme dispositif temporaire, la télévision comme lieu de diffusion et d'exposition de l'art vidéo, le livre d'artiste, ou pour prendre un exemple contemporain, la diffusion d'œuvres d'art numériques qui fait fi de l'architecture et de l'espace muséal - ces exemples invalidant toute velléité de concevoir l'exposition comme objet fixe et immuable ${ }^{28}$. L'exposition redéfinie comme médium doit être repensée selon cette transversalité, irréductible aussi bien à sa structure physique, architecturale, spatiale, qu'à sa simple fonction: il s'agit de la ressaisir dans sa capacité à redéfinir un « sensorium spécifique » et à dépasser « la séparation entre sens et médias ${ }^{29}$ ».

\subsection{La « modulation » muséale : Modèles cybernétiques}

29 La cybernétisation de l'exposition doit être comprise ici comme la conception cybernétique de l'exposition, qui implique son refaçonnage concret (spatial, technique, matériel) par l'intégration de l'idée de feedback, et sa conceptualisation comme système organisé à rétroactions. L'exposition comme dispositif de modulation agit aussi bien sur la médiation entre les objets, les visiteurs, les institutions, que sur les sensibilités attentionnelles des spectateurs.

Nous pouvons étayer notre proposition à travers une série de formulations dues à des curateurs eux-mêmes dont la réception d'auteurs tels que McLuhan ou Wiener a influencé les entreprises de reconceptualisation et de refaçonnage - théorique comme concret - des dispositifs muséaux et expositionnels. C'est notamment au commissaire suédois Pontus Hultén que nous devons l'une des formulations les plus systématiques 
concernant ce qu'il nomme au début des années 1970 (avant qu'il ne devienne le premier directeur du Centre Pompidou) le musée comme "lieu de communication ${ }^{30}$ ». Selon Hultén, ce dernier d'une part est conçu comme le lieu permettant de présenter les recherches artistiques que le musée traditionnel ne peut accueillir, et d'autre part doit prendre acte des «formes de communication » créant des "rapports nouveaux entre l'art et la vie, la vie et la création et la diffusion artistique ", dynamiques nouvelles résultant de Mai 68. "Il s'agissait de favoriser la communication sociale " poursuit Hultén ${ }^{31}$. Plus précisément, ses idées pour ce nouveau type d'institutions sont synthétisées dans un schéma composé de quatre cercles concentriques ${ }^{32}$. La notion d'information est transversale aux quatre cercles, c'est-à-dire que toutes les composantes de ce lieu de communication sont ressaisies à travers la notion d'information. Ce système se précise comme suit :

\section{Information primaire (communication téléprintée)}

2. Espace et outils pour le traitement des informations (ateliers pour le public, les artistes et le personnel du musée)

3. Information traitée (exposition d'art, films, musique, danse, théâtre...)

4. Collection d'art, archives de films... Information traitée et gardée : mémoire ${ }^{33}$.

Dans cette perspective, tout ce qui compose un musée est redéfini comme information, de la plus élémentaire non traitée, à des unités informationnelles transformées en objets culturels et à leur mise en mémoire (rôle de conservation et de collection du musée). Nous pouvons donc parler de cybernétisation du musée et de l'exposition, ces derniers devenant, en conséquence de cette remodélisation, un assemblage régulé par le principe cybernétique de feedback et de régulation de la production et de la circulation de l'information.

À cette schématisation de Hultén, à cette interprétation du musée comme «lieu de communication" réductrice en ce qu'elle ne discrimine pas "communication " et "information", nous pourrions opposer l'approche de la notion de "musée de communication » du curateur argentin Jorge Glusberg. Dans le cadre de ses activités au Centro de Arte y Comunicación (CAYC) de Buenos Aires, Glusberg a proposé une distinction opératoire pour ce nouveau type de musée et d'exposition. Selon lui, la "différence entre un musée de communication et un simple musée d'information repose sur le fait que le premier est orienté vers l'encouragement de la participation tandis que le second ne fait que transmettre du contenu déjà formé dans une structure qui inhibe une telle participation ${ }^{34} »$. En outre, cette notion de participation est l'effet d'une modulation facilitée par l'infrastructure cybernétique, et elle peut être considérée comme l'une des pierres angulaires de cette cybernétisation de l'art, dont le musée et l'institution prennent acte. En effet, dès les années 1960, participation et cybernétisation se font écho: les pratiques artistiques cybernétisées - des " happenings » à l'art informatique émergeant, en passant par les " environnements » et l'art cinétique - étaient considérées comme remettant en question la dialectique spatiale du fond et de la figure, ainsi que le paradigme perspectiviste sur lesquels l'activité spectatorielle classique est fondée, encourageant plutôt des formes de coproduction du sens et d'intelligibilité de l'art (telles que l'interactivité, la collaboration, l'intégration dans l'œuvre). En outre, l'idée de participation renvoie encore au spectre des modes d'engagements corollaires de l'expansion des arts. 

le critique d'art américain Jack Burnham, dans son « Esthétique des systèmes », décrit l'intégration progressive, dans les années 1960, du spectateur dans l'œuvre d'art, les dispositifs communicationnels encourageant l'idée que l'homme est partie intégrante de son environnement, l'art par ordinateur réalisant radicalement cette vision d'une esthétique invalidant la "notion d'un art séparé de son environnement quotidien ${ }^{35}$ ». Aussi, chez Glusberg, la participation s'inscrit dans une vision qui va au-delà d'une conception du musée comme lieu artistique.

La prédominance de l'aspect fonctionnel en art, comme en architecture, étant historiquement un obstacle à la prise en considération adéquate de sa fonction de communication, transcendant considérablement la simple opérationnalité pratique pour devenir un moyen de connexion et d'implication avec le public, un moyen de façonner les relations sociales ${ }^{36}$.

Pour Glusberg et son projet d'une « muséologie critique ", il est nécessaire de réinscrire le musée au-delà du discours artistique pour le replacer dans les canaux qui participent à sa structuration interne et à son effectivité sociale : le musée est fondamentalement un « signe comprenant d'autres signes » et peut donc être désigné par l'expression aux accents mcluhaniens de « musée comme message » (museum-as-message).

Avec le «musée comme signe/message » de Glusberg, le musée devient un médium global incluant d'autres médiums et communiquant lui-même avec d'autres médiums, à l'instar de la ville elle-même. Or, dans l'histoire de la cybernétisation du musée, cette articulation entre ville, exposition et institution a été expérimentée par Peter F. Althaus à la Kunsthalle de Bâle. Sur la base de recherches sur l'espace urbain et sa rationalisation - le développement d'un "modèle de pensée " pour des structures flexibles, capables de croître, de se réduire et de se régénérer permettant l'identification de l'humain avec ce milieu - Althaus a formulé la notion de «musée ouvert », conception éprouvée à travers l'exposition Das offene Museum réalisée en 1970 dans le musée bâlois alors sous sa direction ${ }^{37}$.

Le musée d'art, tout particulièrement, se présente comme un centre d'information et de communication, puisqu'avec l'exemple de l'art - la réaction subjective et immédiate, temporellement et structurellement conditionnée, de l'individu doué d'expression à son milieu - le développement humain de la conscience comme expérience (recherche du passé), confrontation (conflit avec le présent), spéculation (planification de l'avenir) peut être contrôlé. Les processus de perception, d'apprentissage, de création et de transmission doivent être rendus transparents ${ }^{38}$.

\section{Conclusion}

Nous avons défini le médium comme modulateur technique de l'information au sens large, comme ce qui donne sa forme au milieu technique et à notre sensibilité. Nous avons postulé que l'exposition, dispositif technique et informationnel, pouvait être analysée comme médium. Enfin, nous avons suivi son évolution dans les années 1960, qui virent son milieu et son paradigme se transformer.

41

Cette époque voit en effet l'émergence d'une cybernétisation de l'exposition, véritable mutation épistémologique, où se théorise l'exposition comme «lieu de communication ". C'est aussi le moment historique où l'infrastructure technologique rejoint cette nouvelle conceptualisation et la réalise, comme Hultén l'a bien envisagé 
dans son texte «Le musée, lieu de communication»: «Une véritable science de l'information est en train de s'élaborer corrélativement à la nouvelle orientation prise par les sciences et les sciences humaines : histoire de l'art, informatique, cybernétique, linguistique et sémiologie, remise en cause de concepts de théorie, d'histoire, d'espace et de temps, de signe ${ }^{39}$ ». Ces deux niveaux de cybernétisation - institutionnel et informationnel - ne sont pas séparés mais sont les deux faces d'une même pièce.

$\mathrm{Au}$ niveau institutionnel, on voit que l'exposition est de plus en plus intégrée aux recherches universitaires tout en étant populaire auprès du public. L'œuvre d'art selon ce paradigme n'est plus un objet passif, mais selon Hultén, plutôt un événement foisonnant d'informations, de débats et de dialogues ${ }^{40}$. Est rassemblé tout un corpus d'informations liées (critique, médias, information publique) que Glusberg désigne sous le terme générique de " paramédia ${ }^{41}$ ».

$\mathrm{Au}$ niveau informationnel, le musée lui-même, comme c'est le cas avec le Centre Pompidou, devient « une machine en mouvement » selon l'expression de son architecte Renzo Piano ${ }^{42}$. L'usage des dispositifs technologiques devient de plus en plus important dans les expositions qui possèdent une grande capacité à moduler les signaux et à mettre en œuvre de nouveaux circuits d'information. Et il ne s'agit pas simplement d'usage de la technologie, mais plutôt de la pensée cybernétique en tant que paradigme épistémologique qui comprend l'exposition comme un jeu de communication et de signaux.

\section{BIBLIOGRAPHIE}

Alberro Alexander et Stimson Blake (dir.), Institutional Critique. An Anthology of Artists' Writings, MIT Press, 2011.

Althaus Peter F., « Das offene Museum - die Stadt », Kunstnachrichten, No. 6(2), Octobre 1969

Althaus Peter F., « Kommunikation im 'Offenen Museum' », SWB-Kommentare, no 16, September 1970.

Bovier François et Mey Adeena (dir.), Exhibiting the Moving Image. History Revisited, Zurich, JRP-Ringier, 2015.

Burnham Jack, "The Aesthetics of Intelligent Systems », dans The Guggenheim Museum, On the Future of Art, New York, Viking Compass, 1970.

Canguilhem Georges, « Le vivant et son milieu », dans La connaissance de la vie, Paris, Vrin, 2009. Canguilhem Georges, Le normal et le pathologique, 12e éd., Paris, PUF, coll. « Quadrige », 2013.

Deleuze Gilles, «La Peinture et la question des concepts transcription », Cours 17 du 05/05/81, dans La voix de Gilles Deleuze en ligne, http://www2.univ-paris8.fr/deleuze/article.php3? id_article $=83$

Deleuze Gilles, « La Peinture », Cours 18 du 12/05/1981, dans La voix de Gilles Deleuze en ligne, http://www2.univ-paris8.fr/deleuze/article.php3?id_article=198 
Ferguson Bruce W., « Exhibition Rhetorics », dans Reesa Greenberg, Ferguson Bruce and Nairne Sandy (dir.), Thinking About Exhibitions, London and New York, Routledge,1996

Glusberg Jorge, Cool Museums and Hot Museums, Buenos Aires, CAYC, 1980.

Higgins Dick, « Intermedia », The Something Else Newsletter, vol. 1, nº 1, 1966.

Hui Yuk, « Simondon et la question de l'information », Cahiers Simondon, no 6, p. 29-46.

Hui Yuk, « Modulation after Control », new formations: a journal of culture/theory/politics, vol. 84-85, 2015.

Hultén Pontus, « Le musée, lieu de communication », Skira annuel, no 75, 1975.

Krajewski Pascal, « Qu'appelle-t-on un médium ?», Appareil [en ligne], Articles, 11 Février 2015, http://appareil.revues.org/2152

Krauss Rosalind, «A Voyage on the North Sea »: Art in the Age of the Post-medium Condition, London, Thames and Hudson, 2000.

Kravagna Christian (dir.), The Museum as Arena. Artists on Institutional Critique, Kunsthaus Bregenz, Walther König, 2001.

Le Blanc Guillaume, Canguilhem et la vie humaine, Paris, PUF, 2010

Lippard Lucy, Six Years. The Dematerialization of the Art Object from 1966 to 1972, Los Angeles, University of California Press, (1973) 1997.

Marx Werner, The Meaning of Aristotle's "Ontology", Den Haag, 1954.

McNamara Andrew et Ross Toni, « On medium specificity and discipline crossovers in modern art. Jacques Rancière interviewed by Andrew McNamara and Toni Ross ", Australian and New Zealand Journal of Art, 2007, 8(1).

O'Neill Paul, The culture of curating and the curating of culture(s): the development of contemporary curatorial discourse in Europe and North America since 1987 [Thèse], Middlesex University, School of Art and Design, 2007.

Pavie Yann, « Vers le musée du futur: entretien avec Pontus Hultén », Opus International, no 24-25, 1971.

Rancière Jacques, Le partage du sensible. Esthétique et politique, Paris, La Fabrique, 2000

Rancière Jacques, Le Spectateur émancipé, Paris, La Fabrique, 2008.

Schmidt-Burkhardt Astrit, Maciunas' "Learning Machines": From Art History to a Chronology of Fluxus, Vienna / New York, Springer, 2003.

Sicard Monique, «Du médium au milieu », Livraisons de l'histoire de l'architecture [En ligne], 20 | 2010, 10 décembre 2012.

Simondon Gilbert, L'individuation à la lumière des notions de forme et d'information, Grenoble, Editions Jérôme Millon, 2006.

Simondon Gilbert, Communication et information, Paris, Les éditions de transparence, 2010.

Simondon Gilbert, «Culture et technique », dans Sur la technique, Paris, PUF, 2014.

Wallenstein Sven-Olov, « Institutional Desires », dans Möntmann Nina (dir.), Art and Its Institutions, London, Black Dog Publishing, 2006. 


\section{NOTES}

25. Voir notamment : Jacques Rancière, Le Spectateur émancipé, Paris, La Fabrique, 2008.

1. Krajewski Pascal, «Qu'appelle-t-on un médium ? ", Appareil [En ligne], Articles, mis en ligne le 11 février 2015, consulté le 07 juin 2017. URL : http://appareil.revues.org/2152

2. Ibid., p. 3.

3. Ibid., p. 3

4. Sicard Monique, « Du médium au milieu », Livraisons de l'histoire de l'architecture [En ligne], 20 | 2010, 10 décembre 2012, http://lha.revues.org/253 ; DOI : 10.4000/lha.253

5. Le mot "milieu » en français est très lâche et mal-défini. Au $\mathrm{xx}^{\mathrm{e}}$ siècle, les philosophes ont employé le concept de milieu pour traduire des termes étrangers comme l'Umwelt allemand, ainsi que le Fûdo japonais (Augustin Berque). Cependant, ces deux termes véhiculent des sens fort différents. Le premier signifie un monde intériorisé par le sujet, le dernier renvoie à un environnement extérieur ou au climat.

6. Nous renvoyons ici à Aristote. Dans le livre $\mathrm{Z}$ de sa Métaphysique, Aristote donne à la forme (eidos) seule (la forme comprenant elle-même la matière et sa composition) le statut de substance. Voir Aristote, Métaphysique, Traduction (éd. de 1953) de J. Tricot (1893-1963), Éditions Les Échos du Maquis; cette forme-là présente une certaine similitude avec l'autre forme (morphé du l'hylémorphisme). Sur la relation entre les deux terme, voir Werner Marx, The Meaning of Aristotle's "Ontology", Den Haag, 1954.

7. Sur cette question et une comparaison avec les cybernéticiens, voir : Hui Yuk, « Simondon et la question de l'information », Cahiers Simondon, $\mathrm{n}^{\circ}$ 6, Paris, L'Harmattan, p. 29-46.

8. Simondon Gilbert, L'individuation à la lumière des notions de forme et d'information, Grenoble, Éditions Jérôme Millon, 2006, p. 35.

9. De même que Jean-François Lyotard parle d'une heuristique - c'est-à-dire une opération presque algorithmique - dans son analyse du sublime dans la troisième critique de Kant.

10. Simondon Gilbert, Communication et information, Paris, Les éditions de transparence, 2010, p. 159

11. Voir l'analyse de Yuk Hui, « Modulation after Control », new formations: a journal of culture/ theory/politics, vol. 84-85, 2015, p. 74-91.

12. Deleuze Gilles, "La Peinture et la question des concepts transcription», Cours $17 \mathrm{du}$ 05/05/81, dans La voix de Gilles Deleuze en ligne, http://www2.univ-paris8.fr/deleuze/article.php3? id_article $=83$.

13. Deleuze Gilles, "La Peinture », Cours 18 du 12/05/1981, dans La voix de Gilles Deleuze en ligne, http://www2.univ-paris8.fr/deleuze/article.php3?id_article=198.

14. Nous empruntons cette notion à Jacques Rancière. Voir Le partage du sensible. Esthétique et politique, Paris, La Fabrique, 2000.

15. Canguilhem Georges, "Le vivant et son milieu », dans La connaissance de la vie, Paris, Vrin, 2009, p. 165.

16. Le Blanc Guillaume, Canguilhem et la vie humaine, Paris, PUF, 2010, p. 232.

17. Ibid., p. 236.

18. Canguilhem Georges, Le normal et le pathologique, $12^{e}$ éd., Paris, PUF, coll. « Quadrige », 2013, p. 154.

19. Simondon Gilbert, «Culture et technique », dans Sur la technique, Paris, PUF, 2014.

20. Lippard Lucy, Six Years. The Dematerialization of the Art Object from 1966 to 1972, Los Angeles, University of California Press, 1973, 1997.

21. Higgins Dick, "Intermedia", The Something Else Newsletter, vol. 1, $\mathrm{n}^{\circ} 1,1966$.

22. Krauss Rosalind, "A Voyage on the North Sea" in Art in the Age of the Post-medium Condition, Londres, Thames and Hudson, 2000. 
23. Pour une reproduction de ce diagramme voir : Astrit Schmidt-Burkhardt, Maciunas' "Learning Machines": From Art History to a Chronology of Fluxus, Vienne / New York, Springer, 2003, p. 18 sq.

24. Pour ne citer que cet exemple, les pratiques que ce terme recouvre occupent un espace ouvert, d'un côté, par l'héritage duchampien et l'attention portée au contexte d'exposition afin de montrer la condition nominaliste de l'art, et de l'autre, des modalités de critiques idéologiques et sociales du système artistique émergeant en opposition à la guerre du Vietnam. Voir notamment Christian Kravagna (dir.), The Museum as Arena. Artists on Institutional Critique, Kunsthaus Bregenz, Walther König, 2001 ; Alexander Alberro et Blake Stimson (dir.), Institutional Critique. An Anthology of Artists' Writings, MIT Press, 2011.

26. Wallenstein Sven-Olov, «Institutional Desires", dans Möntmann Nina (dir.), Art and Its Institutions, Londres, Black Dog Publishing, 2006, p. 122. Notre traduction.

27. Ferguson Bruce W., "Exhibition Rhetorics", dans Reesa Greenberg, Ferguson Bruce and Nairne Sandy (dir.), Thinking About Exhibitions, Londres et New York, Routledge, 1996, p. 178. Cité dans O'Neill Paul, The Culture of curating and the curating of culture(s): the development of contemporary curatorial discourse in Europe and North America since 1987 [Thèse], Middlesex University, School of Art and Design, 2007, p. 107. Notre traduction.

28. Pour un panorama des modalités diverses d'exposition, du film d'artiste et de l'image en mouvement plus spécifiquement, voir Bovier François et Mey Adeena, « On Moving Images, from the "Exhibition Format" to Public Space ", dans Bovier François et Mey Adeena (dir.), Exhibiting the Moving Image. History Revisited, Zurich, JRP-Ringier, 2015, p. 4-17.

29. McNamara Andrew et Ross Toni, « On medium specificity and discipline crossovers in modern art. Jacques Rancière interviewed by Andrew McNamara and Toni Ross ", Australian and New Zealand Journal of Art, 2007, 8(1), p. 86.

30. Pontus Hultén, « Le musée, lieu de communication », Skira annuel, nº 75, 1975, p. 126.

31. Id.

32. «Un modèle abstrait» du Moderna Museet dont il fut le directeur avant de diriger Beaubourg.

33. Pavie Yann, «Vers le musée du futur : entretien avec Pontus Hultén », Opus International, $\mathrm{n}^{\circ}$ 24-25, 1971, p. 58-61.

34. Glusberg Jorge, Cool Museums and Hot Museums, Buenos Aires, CAYC, 1980, p. 17. Notre traduction.

35. Voir par exemple: Burnham Jack, "The Aesthetics of Intelligent Systems ", dans The Guggenheim Museum, On the Future of Art, New York, Viking Compass, 1970, p. 95-122.

36. Ibid., p. 13.

37. Althaus Peter F., «Das offene Museum - die Stadt », Kunstnachrichten, $n^{\circ} 6(2)$, octobre 1969, non paginé. Notre traduction.

38. Althaus Peter F., «Kommunikation im 'Offenen Museum'», SWB-Kommentare, $\mathrm{n}^{\circ} 16$, September 1970, p. 617.

39. Hultén Pontus, «Le musée, lieu de communication », Skira annuel, nº 75, 1975, p. 126.

40. «Information, dialogues, débats doivent restituer l'œuvre d'art, la remettre au niveau de la vie et non plus en faire l'objet d'un culte passif » (ibid.)

41. Glusberg Jorge, Cool Museums and Hot Museums, Buenos Aires, CAYC, 1980, p. 14.

42. Cité dans Glusberg Jorge, Cool Museums and Hot Museums, Buenos Aires, CAYC, 1980, p. 15. 


\section{RÉSUMÉS}

En se basant sur le moment critique de la cybernétisation de l'institution muséale et de l'exposition, cet article propose de revenir sur la conception de l'exposition comme médium. Il s'agit à la fois de repenser la notion de médium elle-même, en l'articulant avec à la notion de milieu (Canguilhem), et de la redéfinir de façon non-substantialiste comme modulation, à partir des travaux de Simondon et Deleuze. Ceci permet d'élaborer une généalogie de l'exposition comme médium et de retracer le moment historique de son évolution par rapport à l'infrastructure institutionnelle et informationnelle, dans les années 1960.

L'exposition fait partie d'un dispositif institutionnel qui ne touche plus simplement les œuvres d'art et les spectateurs. L'idéal moderniste d'auto-réflexivité du médium va venir toucher l'agencement discursif, sémiotique et matériel qu'est l'exposition, devenue ce que Rancière nomme un "dispositif critique », notant que l'émancipation esthétique se double alors d'une revendication d'émancipation sociale ${ }^{25}$. En effet, nous trouvons d'un côté des stratégies de réflexivité vis-à-vis de l'institution, qui déploient leur charge critique à l'encontre du White cube (comme médium, il est considéré comme un vecteur idéologique, ou pire, il exerce, à travers ses qualités structurantes, une force disciplinaire sur les agents humains et non-humains qui circulent en son sein) ; de l'autre, cet éthos critique est endossé par l'institution elle-même dans la mesure où durant ce moment significatif des années 1960-1970, elle se transforme en ingérant les pratiques et postures artistiques et sociales de résistance ou de subversion à son endroit. Si ce moment signale de nouvelles démarcations et peut être analysé, dans la perspective de l'histoire de l'art, sur le mode d'une rupture, ces deux positions reposent en fin de compte sur le même socle épistémologique : l'affranchissement face au musée et sa démocratisation comme antiillusionnisme, dévoilement. Le sujet éclairé est devenu capable d'échapper à ces vieilles lunes, et s'est équipé pour les dénoncer. Mais comme Sven-Olov Wallenstein l'a justement remarqué à propos de ce désir d'être en dehors de l'institution (artistique mais également psychiatrique) :

By going back to the critical moment of the cybernetisation of the museum and of the exhibition, this essay aims to put forward a thesis considering exhibition as medium. It elaborates on the notion of medium by contrasting it with Canguilhem's notion of milieu, in order to redefine it, in a non-substantialist way, as modulation in light of Simondon's and Deleuze's works. It proposes a genealogy of the exhibition as medium and describes a critical historical trajectory shaped by institutional and informational infrastructures.

INDEX

Mots-clés : milieu, information, musée, communication, Canguilhem, Simondon, Hultén

\section{AUTEURS}

\section{YUK HUI}

Docteur en philosophie, enseignant-chercheur à l'Université de Leuphana en Allemagne. Il a dirigé l'anthologie 30 Years after Les Immatériaux : Art, Science and Theory (Meson Press, 2015) dédiée à l'exposition de Lyotard de 1985. Il est l'auteur de On the Existence of Digital Objects (University of Minessota Press, 2016) et de The Question Concerning Technology in China. An Essay in Cosmotechniques (Urbanomic, 2016) yuk.hui@leuphana.de 


\section{ADEENA MEY}

Critique d'art et doctorant à la section d'histoire et d'esthétique du cinéma de l'Université de Lausanne en Suisse. Avec François Bovier il a dirigé : Exhibiting the Moving Image. History Revisited (Zurich, JRP-Ringier, 2015) ; Cinema in the Expanded Field (Zurich, JRP-Ringier, 2015) ; René Berger. L'art vidéo (Zurich, JRP-Ringier, 2014) ; Cinéma Exposé/Exhibited Cinema (ECAL/Les presses du réel, 2015, adeena.mey@unil.ch 